\title{
Performance and results of the high-resolution biogeochemical model PELAGOS025 v1.0 within NEMO v3.4
}

\author{
Italo Epicoco ${ }^{1,2}$, Silvia Mocavero ${ }^{2}$, Francesca Macchia ${ }^{2}$, Marcello Vichi ${ }^{3}$, Tomas Lovato ${ }^{2}$ Simona Masina $^{2}$, and \\ Giovanni Aloisio ${ }^{1,2}$ \\ ${ }^{1}$ Department of Innovation Engineering, University of Salento, via per Monteroni, 73100 Lecce, Italy \\ ${ }^{2}$ Euro-Mediterranean Centre on Climate Change Foundation, via Augusto Imperatore 16, 73100 Lecce, Italy \\ ${ }^{3}$ Department of Oceanography, University of Cape Town, Cape Town, South Africa
}

Correspondence to: Italo Epicoco (italo.epicoco@unisalento.it)

Received: 3 November 2015 - Published in Geosci. Model Dev. Discuss.: 16 December 2015

Revised: 1 April 2016 - Accepted: 10 May 2016 - Published: 10 June 2016

\begin{abstract}
The present work aims at evaluating the scalability performance of a high-resolution global ocean biogeochemistry model (PELAGOS025) on massive parallel architectures and the benefits in terms of the time-to-solution reduction. PELAGOS025 is an on-line coupling between the Nucleus for the European Modelling of the Ocean (NEMO) physical ocean model and the Biogeochemical Flux Model (BFM) biogeochemical model. Both the models use a parallel domain decomposition along the horizontal dimension. The parallelisation is based on the message passing paradigm. The performance analysis has been done on two parallel architectures, an IBM BlueGene/Q at ALCF (Argonne Leadership Computing Facilities) and an IBM iDataPlex with Sandy Bridge processors at the CMCC (Euro Mediterranean Center on Climate Change). The outcome of the analysis demonstrated that the lack of scalability is due to several factors such as the I/O operations, the memory contention, the load unbalancing due to the memory structure of the BFM component and, for the BlueGene/Q, the absence of a hybrid parallelisation approach.
\end{abstract}

\section{Introduction}

Nowadays, the study of climate change needs high-resolution simulations as one of the possible strategies to reduce uncertainty in climate predictions. In addition, the interaction of the physical components of the climate system with Earth biogeochemistry and socio-economical aspects implies that multiple dynamical models are coupled together in the socalled Earth system models (Schellnhuber, 1999; Claussen, 2000), increasing the complexity of the software tool. Nextgeneration leadership class computing systems can be considered a deep revolution in climate change applications (Dongarra et al., 2011), allowing ever higher resolutions of climate models that will match or even surpass the resolution of today's operational weather forecast models. In particular, exascale will be able to provide the computational resources needed to increase resolution and complexity as required (Washington, 2005). However, climate and Earth system simulations can benefit from exascale as long as the models are capable of scaling their performances. There are several issues to be considered when scaling models to reach a performance up to an order of $10^{18}$ floating point operations per second (Washington, 2008). At higher resolution, new physical aspects must be taken into account and integrated into the climate models (see e.g. Siedler et al., 2013); it is necessary to design scalable computational kernels and algorithms, as well as to consider new approaches and paradigms in the parallel programming, in order to follow the features of the exaflops architectures. Often, to exploit the exascale potentiality, the so-called "legacy" climate models require a deep re-engineering, like e.g. the improvement of the computational kernels, new parallel approaches and new scalable algorithms. Moreover, new models, dynamic grids and new numerical solvers have to be conceived on exascale computers to carry out efficient operations.

The community climate models have to be carefully analysed in order to emphasise the scalability bottlenecks, which 
could not be the same on different architectures. Moreover, the implemented parallel approaches and the available alternatives have to be investigated to select the best strategy. The computational scientists have to decide whether the model has to be re-designed from scratch or whether it can be optimised in order to exploit the next-generation architectures. The performance could be improved by using optimised numerical libraries (Dongarra et al., 1988, 1990; Blackford et al., 1996; Balay et al., 1997) or using tools to improve the I/O operations (XIOS, 2013; Balaji et al., 2013). In any case, the first required step is the analysis of the model scalability on (as many as possible) multiple architectures for testing the behaviour on heterogeneous resources. Dennis and Loft (2011) stressed the importance of testing the weak scalability by studying the impact of increasing both the resolution and core counts by factors of 10 to 100 using the Community Climate System Model (CCSM). Several issues related to the common code design and implementation emerged. This prevented the efficient execution of these applications on very large core counts. Worley et al. (2011) described the performance engineering aspects of the Community Earth System Model (CESM) and reported the performance scaling on both the Cray XT5 and the IBM BG/P for four representative production simulations, by varying both the problem size and the included physical processes. The bottleneck can be a particular kernel of the model or a particular operation, such as the $\mathrm{I} / \mathrm{O}$, or an entire model component within a coupled model, which is likely to be rather common with coupled Earth system models. The scalability of a coupled model can be improved by balancing the model component load (Epicoco et al., 2011) or optimising the component that limits the performance. Mirin and Worley (2012) identified the CESM atmosphere component (CAM) as the most computationally expensive. The improvement in the CAM performance scalability can be achieved by means of new optimised communication protocols, and through the reduction of the computational bottlenecks.

As an example of this assessment of multi-component Earth system models, we focused on an implementation that is likely to be standard in the next generation of climate models. We considered two components that are usually computationally demanding, the ocean physics and ocean biogeochemistry. As in most of the cases, ocean biogeochemical models are tightly linked to the ocean physics computational cores, as they share the same grid and numerical schemes. In particular, the present work aims at analysing the computational performance of the Nucleus for the European Modelling of the Ocean (NEMO) oceanic model at $0.25^{\circ}$ horizontal resolution coupled with the Biogeochemical Flux Model (BFM). The paper is organised as follows: the next section introduces the coupled model and the experimental set-up, Sect. 3 shows the main results in terms of strong scalability of the model, Sect. 4 describes the methodology used for the code profiling, focusing on two different architectures, Sect. 5 discusses the data structures used in NEMO and in the BFM and highlights pros and cons, Sect. 6 illustrates the memory allocation model, and the last section ends with some conclusions and future perspectives.

\section{The PELAGOS025 biogeochemical model}

PELAGOS (PELAgic biogeochemistry for Global Ocean Simulations; Vichi et al., 2007; Vichi and Masina, 2009) is a coupling between the NEMO general circulation model (version 3.4, http://www.nemo-ocean.eu) and the Biogeochemical Flux Model (BFM, version 5, http:// bfm-community.eu). The BFM is based on a biomass continuum description of the lower trophic levels of the marine system. The model is meant to describe the planktonic ecosystem in the global ocean; therefore, it complements the classical ocean carbon cycle equations with the fluxes of nutrients (nitrogen, phosphorus, silicate and iron) among multiple biological functional groups, namely phytoplankton, zooplankton and bacteria. From a computational point of view, the use of multiple chemical constituents to represent the functional groups implies the implementation of several state variables that are about 2 to 3 times larger than the standard carbon cycle models (this current formulation has 52 state variables; see Vichi et al., 2015a, for a description of the equations). In addition, the model is capable of storing all the rates of transfer of the constituents among the functional groups, which adds substantially to the computational load.

The coupling between NEMO and the BFM occurs at every time step and each processing element (PE) resolves the integration of both physical and biogeochemical model equations. The memory layout of the BFM can be defined by construction as zero- or one-dimensional, and the latter is used for the coupling with NEMO by considering only the ocean points of the model subdomain. This implies that each BFM variable is a one-dimensional array, with all the land points stripped out from the three-dimensional domain of NEMO, and the remapping into the ocean grid is done only when dealing with transport processes. This operation is done for every subdomain of the grid decomposition. A thorough description of the NEMO-BFM coupling is detailed in Vichi et al. (2015b), publicly available on the BFM website.

NEMO uses a horizontal domain decomposition based on a pure MPI (message passing interface) approach. Once the number of cores has been chosen, the number of subdomains along the two horizontal directions (hereinafter jpni and jpnj) are consequently defined. The numerical discretisation used in NEMO is based on finite differences. According to this method, the communication pattern among the parallel tasks is based on the five-point cross stencil. The best decomposition strategy for reducing the communication overhead is to select jpni and jpnj to obtain subdomains as square as possible. By following this procedure, the communication overhead is minimal. However, coupling the biogeochemical component, the number of ocean points for 
each subdomain becomes a crucial factor, since the BFM, unlike NEMO, performs the computation only on these points. A pre-processing tool has been written to establish the best domain decomposition that minimises the number of ocean points of the biggest subdomain. In addition, a NEMO feature allows one to exclude the domains with land points only. Reid (2009) demonstrated that the removal of land processes reduces the resource usage by up to $25 \%$ and also gives a small reduction in the total runtime. The subdomains to be excluded depend on the bathymetry. Figure 1 shows a domain decomposition with the bathymetry in the background highlighting those subdomains excluded from the computation because they are made up of land points only.

\section{Performance analysis}

\subsection{Test case}

The PELAGOS model was tested in this work at the highest available horizontal resolution of $0.25^{\circ}$ described in McKiver et al. (2015), where all the details of the simulation setup can be found. PELAGOS025 is a configuration based on the ORCA025 grid $(1442 \times 1021$ grid points in the horizontal with 50 vertical levels), going from an effective resolution of $28 \mathrm{~km}$ at the Equator to $10 \mathrm{~km}$ at the poles (Barnier et al., 2006). A time step of $18 \mathrm{~min}$ is used both for the physical and biogeochemical model, while the sea ice model is called every five steps. For each run we simulated 1 day with a total of 80 time steps. This specific experiment focused more on computational performances and less on the I/O behaviour because, at the time of the experimental analysis, it was possible to use an I/O strategy where each process wrote its own outputs and restart files. When the number of cores increases beyond 2048, the number of files cannot be efficiently handled by the file system. Further experiments will be performed using the XIOS (XIOS, 2013) library that will be supported by version 3.6 of NEMO.

The analysis of the strong scalability of the code has been performed on two architectures: the first one is a BlueGene/Q (named VESTA), located at the Argonne Leadership Computing Facilities (ALCF/ANL); the second one is the ATHENA system, available at the CMCC (Euro Mediterranean Center on Climate Change), an iDataPlex equipped with Intel Sandy Bridge processors. The activity has been conducted in collaboration with the ALCF/ANL. Details about the systems are reported in Table 1. The main differences among the machines are the number of hardware threads. VESTA can handle simultaneous multithreading (SMT) up to four threads, while the Sandy Bridge architecture supports the execution of two threads simultaneously. Even if ATHENA has a higher value of the peak performance per node, VESTA is a very high scalable architecture. Finally, the communication network is different: BG/Q uses a Torus network with five dimensions; it is characterised by

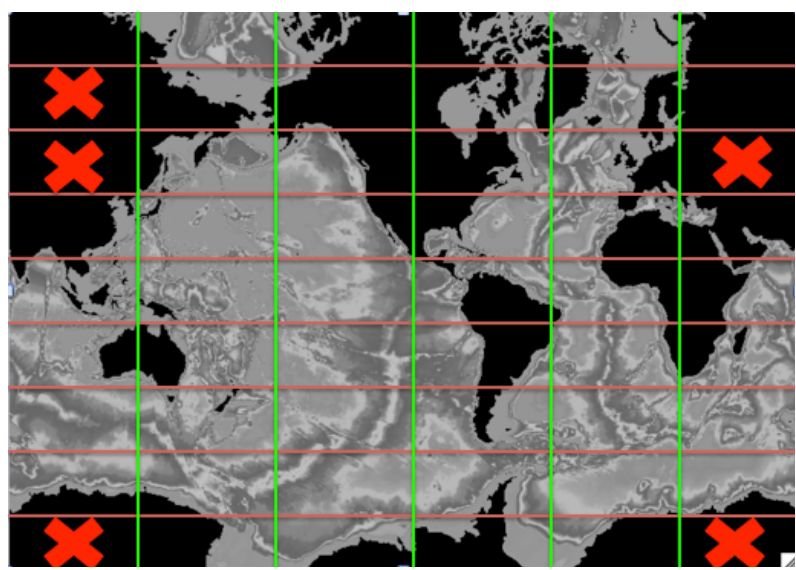

Figure 1. Example of PELAGOS025 decomposition on 54 subdomains. There are five subdomains with land points only (marked by an $\mathrm{X}$ ). These subdomains are not included in the computation.

Table 1. Architecture parameters related to the BlueGene/Q (named VESTA), located at the Argonne Leadership Computing Facilities (ALCF/ANL) and the iDataPlex equipped with Intel Sandy Bridge processors (named ATHENA), located at the CMCC.

\begin{tabular}{lll}
\hline Design parameters & BG/Q (ANL) & IBM iDataPlex (CMCC) \\
\hline Processor & PowerPC A2 & Intel Xeon Sandy Bridge \\
Cores/node & 16 & 16 \\
Hardware threads & 4 & 2 \\
Flop/clock/core & 8 & 8 \\
Flop/node (GFlop) & 204.8 & 332.8 \\
Clock speed (GHz) & 1.6 & 2.6 \\
RAM/core (GB) & 1 & 4 \\
Network & 5-D Torus & Infiniband 4×FDR \\
\hline
\end{tabular}

several partitions made up of 32 up to 1024 nodes. During the execution, an entire partition is reserved for the job. This means that the job acquires the use of both the nodes and the network partition exclusively. The ATHENA nodes are connected through an infiniband switch that is shared among all the running jobs.

Table 2 reports the considered domain decomposition corresponding to the selected number of cores on ATHENA and VESTA machines. The table also contains the number of nodes used for each experiment. SMT has not been used on either machine. NEMO being a memory-intensive application, the use of SMT does not produce major improvements in the performance; notably, performance can even deteriorate due to the memory contention produced by the simultaneous execution of the threads. Each experiment has been repeated five times, with a total of 30 runs on ATHENA and 20 on VESTA. 
Table 2. Domain decompositions used for the experiments on the Sandy Bridge (ATHENA) and BG/Q (VESTA) architectures. The first two columns report the number of subdomains along the two horizontal directions; the third column shows the total number of processes excluding the land ones. A column follows indicating the number of nodes used to run the experiment, while the last columns show the average execution time, in s, for a time step of the simulation on both machines.

\begin{tabular}{lrrrrr}
\hline jpni & jpnj & jpnij & nodes & SB s step $^{-1}$ & BG/Q s step $^{-1}$ \\
\hline 6 & 29 & 160 & 10 & 25.05 & - \\
38 & 18 & 544 & 34 & 7.42 & - \\
52 & 24 & 944 & 59 & 5.27 & - \\
104 & 17 & 1344 & 84 & 4.78 & - \\
70 & 34 & 1728 & 108 & 3.19 & - \\
122 & 23 & 2048 & 128 & 3.27 & 16.25 \\
363 & 15 & 4096 & 256 & - & 10.81 \\
281 & 42 & 8192 & 512 & - & 8.40 \\
149 & 166 & 16384 & 1024 & - & 7.15 \\
\hline
\end{tabular}

\subsection{Strong scalability}

The performance analysis started from the evaluation of the parallel scalability. Two definitions of parallel scalability can be considered: the strong and weak scalability. The former is defined as the computational behaviour of the application when the number of computing elements increases for a fixed problem size; the latter describes how the execution time changes with the number of computing elements for a fixed grain size. This means that the computational work assigned to each processor is fixed, and hence the problem size grows with the number of processes. The weak scalability is relevant when a parallel architecture is used for solving problems with a variable size, and the main goal is to improve the solution accuracy rather than to reduce the time-to-solution. The strong scalability is relevant for applications with a fixed problem size, and hence the parallel architecture is used to reduce the time-to-solution. The PELAGOS025 coupled model can be considered a problem with a fixed size and the main goal is to use computational power to reduce the time-tosolution.

The charts in Figs. 2, 3 and 4 show the scalability results respectively in terms of speedup, execution time and SYPD (simulated years per day), a metric for measuring the simulation throughput usually referred to by climate scientists to evaluate the model performance (see e.g. Parashar et al., 2010). On the ATHENA cluster, the tests have been executed up to 2048 cores. Figure 3 shows that the execution time on 2048 cores increases with respect to the run on 1728 cores, which indicates a lack of scalability. For this reason the analysis on ATHENA was limited to 2048 cores. On the VESTA machine the analysis has been performed on up to 16384 cores. Even if there is a factor of 10 between the resources used on the two machines, the best execution time obtained on the Sandy Bridge architecture is halved with

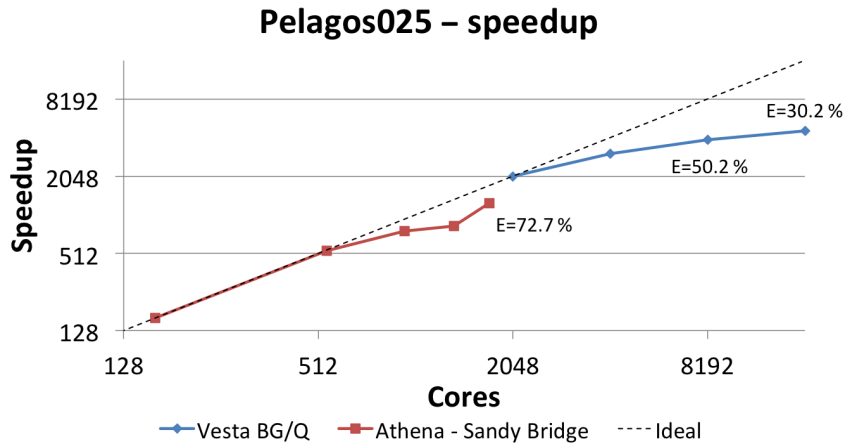

Figure 2. Scalability of the PELAGOS025 configuration: comparison between the results obtained on ATHENA and VESTA. The red line represents the speedup of the model on ATHENA, the blue line on VESTA. The dashed line represents the ideal speedup.

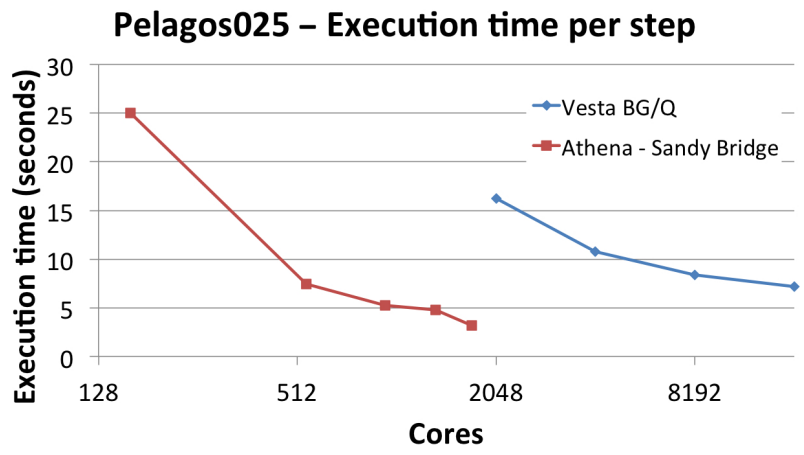

Figure 3. Scalability of the PELAGOS025 configuration: comparison between the results obtained on ATHENA and VESTA. The red line represents the execution time for a time step of the model on ATHENA, the blue line on VESTA.

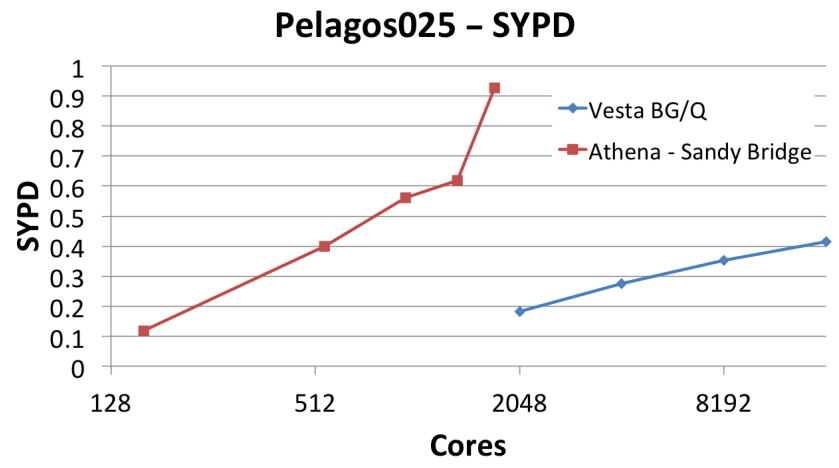

Figure 4. Scalability of the PELAGOS025 configuration: comparison between the results obtained on ATHENA and VESTA. The red line represents the simulated years per day of the model on ATHENA, the blue line on VESTA. 
respect to the BG/Q. The decrease in scalability calls for a deeper analysis of the bottlenecks and the need for a broad optimisation activity.

Figure 5 shows how the MPI communication time tends to decrease with the number of cores. Here only two configurations for each architecture have been considered: with 1344 and 2048 processes on Sandy Bridge and with 2048 and 4096 processes on $\mathrm{BG} / \mathrm{Q}$.

The MPI communication time decreases for two main reasons: the first one relates to the communication type that can be classified as a neighbourhood collective, which means that each process communicates only with its neighbours and no global communication happens, so the number of messages per core does not change when the number of processes increases; the second reason involves the amount of data exchanged between processes, which becomes smaller when the local subdomain shrinks.

\section{Code profiling}

The optimisation process of a code requires the analysis of the bottlenecks that limit the scalability. The investigation methodology used in the present work is based on the analysis at the routine level. Two different reference decompositions have been taken into account and the execution times of the main routines for the two decompositions have been analysed in order to evaluate the speedup of each single routine. The gprof utility has been used to measure the execution time of the PELAGOS routines. The gprof output consists of two parts: the flat profile and the call graph. The flat profile gives the total execution time spent in each function and its percentage of the total running time, providing an easy way to identify the hotspots.

As with many codes in this domain, NEMO has a broad, flat execution profile with no single routine accounting for more than $20 \%$ of the runtime. In a previous work (Epicoco et al., 2014), a detailed analysis of the main code bottlenecks using the roofline model is provided. The profiling data, reported in Table 3, refer to the computationally heaviest process in an execution with 2048 processes. The top 10 routines reported by gprof are the same for both architectures; the difference is in the percentage of the running time. The total computational workload is given by $40 \%$ of NEMO and $60 \%$ of the BFM component. On BG/Q we also observe a significant runtime in two system calls for accessing the input files.

Some of the most time-consuming routines are related to the advection (tra_adv_muscl) and diffusion (tra_ldf_iso and tra_zdf_imp). These routines can also be considered widely representative of the whole NEMO code since their code structure consists of several triply nested loops along the three dimensions of the domain interspersed with halo exchanges among MPI subdomains. In common with the NEMO code as a whole, these tracer-

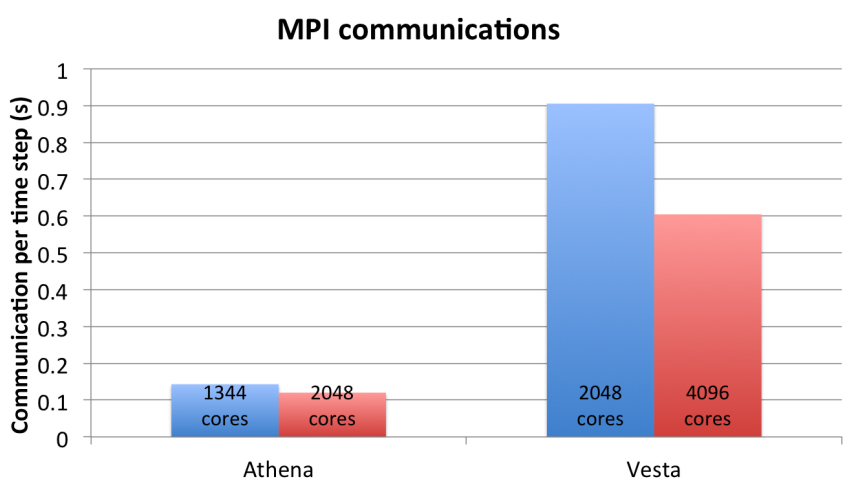

Figure 5. MPI communication time for two configurations for each architecture: with 1344 and 2048 processes on ATHENA and with 2048 and 4096 processes on VESTA.

Table 3. Code profiling on BG/Q and Intel Sandy Bridge. The data have been taken with the gprof tool and they refer to the timing of the most computationally loaded process on a run with 2048 processes.

\begin{tabular}{lrlr}
\hline \multicolumn{2}{c}{ BG/Q - VESTA } & \multicolumn{2}{c}{ Sandy Bridge - ATHENA } \\
Routine & Time (\%) & Routine & Time $(\%)$ \\
\hline calchplus & 7.70 & tra_ldf_iso & 12.36 \\
flux_vector & 7.25 & flux_vector & 11.36 \\
tra_ldf_iso & 4.36 & tra_adv_muscl & 10.16 \\
trc_trp_bfm & 3.96 & trc_trp_bfm & 9.69 \\
tra_adv_muscl & 3.71 & calchplus & 7.99 \\
microzoodynamics & 2.95 & tra_zdf_imp & 7.07 \\
mesozoodynamics & 2.82 & mesozoodynamics & 4.1 \\
tra_zdf_imp & 2.32 & microzoodynamics & 3.79 \\
phytodynamics & 1.48 & pelglobaldynamics & 3.42 \\
calcmean_bfm & 1.20 & phytodynamics & 2.95 \\
pelglobaldynamics & 1.05 & calcmean_bfm & 2.64 \\
_lseek_nocancel & 16.16 & & \\
_read_nocancel & 13.16 & & \\
\hline
\end{tabular}

related kernels are memory-bandwidth bound due to the large number of array accesses required (primarily for reading). This situation is not helped by NEMO's historical development for vector processors since this has encouraged the use of arrays for storing intermediate results. Writing and reading these arrays use up memory bandwidth that in some cases can be saved by simply re-computing the results as required. On the other hand the structure of the NEMO code is suited for the increasingly wide SIMD (single instruction multiple data) unit of the upcoming processors. Also, the BFM presents a code structure suitable for the vector units since it implements a zero-dimensional approach, avoiding any indirect reference to the memory. In any case, with the roofline analysis we demonstrated that BFM routines are characterised by a low arithmetic intensity. It measures the number of operations per byte accessed from the main memory. A low arithmetic intensity also implies that the computa- 
Table 4. Name and description of the routines selected during the code profiling analyses. The routines identified as belonging to the BFM are also the ones that originate from NEMO, but they have been modified for the BFM memory structure.

\begin{tabular}{|c|c|c|}
\hline F90 name & Model & Tasks \\
\hline trc_adv, trc_adv_muscl & NEMO & $\begin{array}{l}\text { Advection of biogeochemical tracers (main caller and specific advection } \\
\text { scheme) }\end{array}$ \\
\hline calchplus, drtsafe 2 & BFM & $\begin{array}{l}\text { Main caller and the iterative scheme to solve the carbonate system equi- } \\
\text { librium using the Newton-Raphson method }\end{array}$ \\
\hline tra_qsr & NEMO & Computation of the temperature trend due to solar radiation penetration \\
\hline div_cur & NEMO & Computation of horizontal divergence and relative vorticity \\
\hline dyn_spg_flt, sol_pcg & NEMO & $\begin{array}{l}\text { Main caller and pre-conditioned conjugate gradient solver for the ellip- } \\
\text { tic differential equation of the surface pressure gradient }\end{array}$ \\
\hline flux_vector & BFM & $\begin{array}{l}\text { Helper routine that stores the fluxes of material between the BFM state } \\
\text { variables }\end{array}$ \\
\hline $\begin{array}{l}\text { tra_ldf_iso, ldf_slp, } \\
\text { dyn_ldf_bilap }\end{array}$ & NEMO & $\begin{array}{l}\text { Horizontal turbulent diffusion for temperature and salinity (along isopy- } \\
\text { cnal levels, with computation of the slope of isopycnals) and momentum }\end{array}$ \\
\hline trc_trp_bfm & BFM & $\begin{array}{l}\text { Main caller to advection-diffusion routines for biogeochemical tracers. } \\
\text { It loops over the number of BFM state variables and does the remapping } \\
\text { between one-dimensional and three-dimensional data structures. }\end{array}$ \\
\hline microzoodynamics & BFM & Computation of the reaction terms for microzooplankton \\
\hline mesozoodynamics & BFM & Computation of the reaction terms for mesozoplankton \\
\hline tra_zdf_imp & NEMO & $\begin{array}{l}\text { Computation of vertical diffusion of temperature and salinity using an } \\
\text { implicit numerical scheme }\end{array}$ \\
\hline phytodynamics & BFM & Computation of the reaction terms for phytoplankton \\
\hline pelglobaldynamics & BFM & $\begin{array}{l}\text { Computation of diagnostic terms used in the pelagic model (chloro- } \\
\text { phyll, nutrient ratios, etc.) }\end{array}$ \\
\hline pelbacdynamics & BFM & Computation of the reaction terms for pelagic bacteria \\
\hline trc_stp & NEMO & $\begin{array}{l}\text { Main caller of the time stepping for biogeochemical tracers. It calls the } \\
\text { BFM routines and the transport of biogeochemical tracers. }\end{array}$ \\
\hline zps_hde & NEMO & $\begin{array}{l}\text { Computation of the bottom horizontal gradient for temperature, salinity } \\
\text { and density when using partial steps }\end{array}$ \\
\hline tra_sbc, sbc & NEMO & Surface boundary conditions for temperature and salinity \\
\hline tra_bbl & NEMO & Bottom boundary layer condition for temperature and salinity \\
\hline
\end{tabular}

tional speed (measured in GFlops) is limited by the memory bandwidth.

In addition, on the BG/Q machine, an in-depth analysis using the Hardware Performance Monitoring (HPM, Lakner et al., 2008) tool has been performed in order to verify the overall intrinsic performance. For reference, a complete description of the code flowchart and naming conventions of the various routines is available in the BFM manuals (Vichi et al., 2015a, b). We report in Table 4 a description of the main tasks performed by the routines that have been identified by the code profiling on the two architectures.

\subsection{BG/Q}

The profiling at routine level helps to discover the model bottlenecks. The code profiling has been performed with 2048 and 4096 cores. The most time-consuming routines have been selected in both cases. Figure 6 shows the speedup for the main identified routines. The speedup is evaluated as the ratio between the execution time on 2048 and 4096 cores, so the ideal value should be 2 . However, none of the routines reached the ideal speedup. This is because the computing time for the BFM strictly depends on the number of ocean points. Starting from the considered decompositions (2048 and 4096), the number of ocean points assigned to the most 
Table 5. Code profiling by applying the HPM (Hardware Performance Monitoring) tool on the BG/Q cluster. The performance values do not include the start-up or the I/O operations. The first column reports the measured parameters, while the other ones show the values on two reference decompositions, respectively, on 2048 and 4096 cores.

\begin{tabular}{|c|c|c|}
\hline Measure & Values on 2048 cores & Values on 4096 cores \\
\hline Instruction mix & $\mathrm{FPU}=4.49 \% ; \mathrm{FXU}=95.51 \%$ & $\mathrm{FPU}=3.01 \% ; \mathrm{FXU}=96.99 \%$ \\
\hline Instructions per cycle/core & 0.2548 & 0.2769 \\
\hline Gflops/node (peak 204) & 0.598 (Gflops) & 0.436 (Gflops) \\
\hline DDR traffic/node & 1.775 (bytes cycle ${ }^{-1}$ ) & 1.168 (bytes cycle ${ }^{-1}$ ) \\
\hline Loads that hit in L1 or L1P & $98.8 \%$ & $99.1 \%$ \\
\hline MPI communication time & $144.84(\mathrm{~s})$ & $96.72(\mathrm{~s})$ \\
\hline Total elapsed time & $397.85(\mathrm{~s})$ & $281.71(\mathrm{~s})$ \\
\hline
\end{tabular}

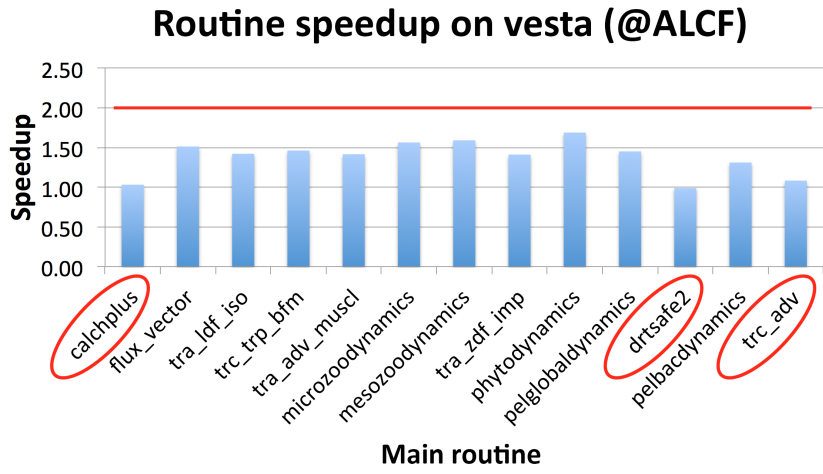

Figure 6. Analysis of scalability of the main routines on the BG/Q cluster in terms of speedup. The speedup is evaluated as the ratio between the execution time on 2048 and 4096 cores. Hence the ideal scalability is reached with speedup equal to 2 . The red circles indicate the routines whose speedup is far from the ideal value.

computationally loaded process is respectively 28553 and 19506. Even if the number of cores has been doubled, the maximum number of ocean points has not been halved. The scalability of the BFM is thus heavily affected by the load balancing problem. Moreover, the three routines, highlighted in Fig. 6 (cf. Table 4), do not scale at all.

Table 5 shows the results obtained by applying the HPM to the BG/Q machine. The performance values refer to the execution of nine time steps (from the second to the tenth) and they do not include the start-up operations or the I/O operations (during the first time step the input data are read while the restart and output writing has been disabled). The instruction mix refers to the ratio between the floating point and the total instructions. The best mix should be $50 \%$. BG/Q has two different and independent pipelines for executing floating point and integer instructions: an instruction on the Floating Point Unit (FPU) can be executed simultaneously with an instruction on the Fixed Point Unit (FXU). The instruction mix is completely unbalanced. However, we have to consider that the FXU includes the load and store instructions to access the memory. Moreover, the execution of a single time step reaches a rate of 0.517 Gflops per node. Considering that one BG/Q core can theoretically execute eight operations per clock cycle, with a frequency of $1.6 \mathrm{GHz}$, a single BG/Q node (which includes 16 cores) can theoretically reach 204.8 Gflops. PELAGOS025 exploits only $0.25 \%$ of the theoretical peak performance. Even if we consider the BG/Q sustained peak performance, as reported in the top500 list (www.top500.org), which is equal to 174.7 Gflops, PELAGOS025 does not reach $0.3 \%$ of the BG/Q sustained peak performance. This means that the model exploits only a very small part of the computational potential of the architecture. There are several reasons which can justify low efficiency: (i) the NEMO code does not exploit the simultaneous multithreading since the parallelisation is based on a pure MPI approach; (ii) a low level of arithmetic intensity which limits the performance to the bandwidth bound; and (iii) a low level of loop vectorisation which does not allow one to properly exploit the SIMD unit. The last consideration regards the percentage of L1 cache hits: the high value means that the memory hierarchy is well exploited.

\subsection{IBM iDataPlex}

The analysis of routine scalability on the iDataPlex architecture has been performed on two other reference decompositions respectively on 1344 and 2048 cores. Figure 7 shows the results in terms of speedup. In this case the data have been obtained from the NEMO profiling tool by activating the nn_timing flag in the configuration file. It provides information at a higher level. Indeed, only the routines directly called from the main loop on time steps are measured. For the considered configurations, the number of ocean points of the most loaded process are respectively 46693 and 30863 , so that the ratio between both the number of ocean points and between the number of cores is about 1.5. Even if this consideration could lead one to think that the balancing algorithm is efficient, unfortunately it is only a coincidence, and this happens only for the considered decompositions: the code does not include an efficient balancing algorithm. With this architecture, there are more routines with a speedup value far from the ideal one, and interestingly they do not correspond to those ones identified in BG/Q. The two consid- 


\section{Routine speedup on Athena (@CMCC)}

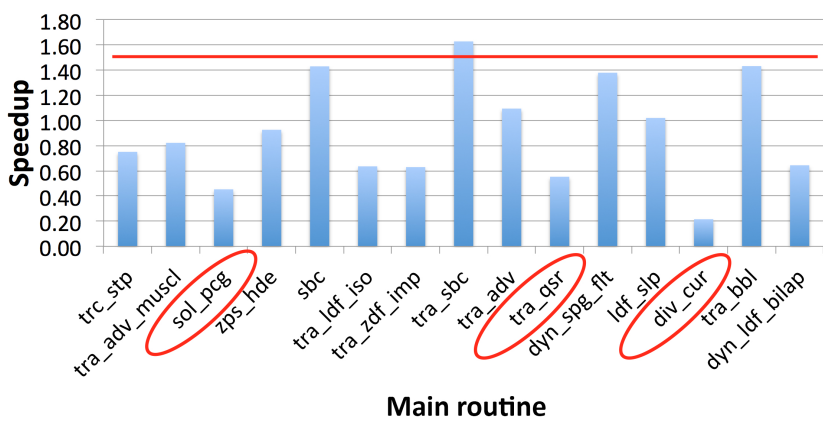

Figure 7. Analysis of scalability of the main routines on the iDataPlex cluster in terms of speedup. The speedup is evaluated as the ratio between the execution time on 1344 and 2048 cores. Hence the ideal scalability is reached with a speedup equal to 1.52 . The red circles indicate the routines whose speedup is far from the ideal value. The data, in this case, have been taken using the NEMO profiling support which provides information at a higher level.

ered architectures deeply differ in terms of processor technology, functional units, computational data path, memory hierarchy, network interconnection and software stack such as compilers and libraries. The BG/Q is based on lightweight processors at $1.6 \mathrm{GHz}$ mainly suited for that part of the code which is computing intensively with massive use of floating point operations and with a high level of arithmetic intensity. Moreover, the optimisations introduced by the compiler are mainly related to the vectorisation level, and this can explain why the routines identified on IBM iDataPlex with a Sandy Bridge processor are different from those ones identified on BG/Q. Further analyses are needed in order to discover the peculiarities of the highlighted routines or the presence of common issues, such as a high communication overhead or a low parallelism level. In this case the performance could be improved by introducing a hybrid parallelisation approach.

\section{NEMO and BFM data structures}

In this section we deeply analyse the differences between the data structures adopted in NEMO and in the BFM, and we evaluate which one is better for use. A three-dimensional matrix data structure is used in NEMO. Each matrix also includes the points over land and it is the natural implementation of the subdomains defined as regular meshes by the finite difference numerical scheme. Even if this data structure brings some overhead due to the computation and memorisation of the points over land, it maintains the topology required by the numerical domain. The finite difference scheme requires each point to be updated considering its six neighbours, establishing a topological relationship among each point in the domain. Using a three-dimensional matrix to implement the numerical scheme, this relationship is maintained, and the topological position of a point in the domain can be directly derived by its three indexes in the matrix. Changing this data structure would imply the adoption of additional information for representing the topology with a negative impact on the performance due to indirect memory references, introduction of cache misses and reduction of the loop vectorisation level.

The BFM uses instead a one-dimensional array data structure with all the land points stripped out from the threedimensional domain. The BFM is zero-dimensional by construction, so the new value of a state variable in a point depends only on the other state variables in the same point, and no relationship among the points is needed. The transport term of the pelagic variables is demanded to NEMO, and this requires a remapping from a one-dimensional to a three-dimensional data structure and vice versa at each coupling step. In this section we aim at evaluating whether the adoption of the three-dimensional matrix data structure for the BFM can improve the performance of the whole model. Three main aspects will be evaluated: the number of floating point operations, the load balancing and the main memory allocation. The evaluation has been conducted by choosing a number of processes that lead each subdomain of the PELAGOS025 configuration to have exactly a square shape. Figure 8 depicts all of the parallel decompositions that satisfy this squared domain condition. When the number of processes $p x$ along $i$ and $p y$ along $j$ fall in the blue region, a squared domain decomposition is generated. The graph has been built considering that in order to obtain a squared domain with just one line for the halo, the following equation must be satisfied:

$$
\left\lceil\frac{\text { iglo-3 }}{\mathrm{px}}\right\rceil=\left\lceil\frac{\text { jglo-3 }}{\mathrm{py}}\right\rceil \text { py, } \mathrm{px} \in \mathbb{N},
$$

where iglo and jglo are the size of the whole domain and px and py are the number of processes to choose. With this choice any effect due to the shape of the domain is eliminated. In the following subsections we analyse the three performance aspects keeping in mind that the aim is to compare the BFM when it adopts a one- or three-dimensional data structure. The analysis is not intended as a comparison between NEMO and the BFM.

\subsection{Number of floating point operations}

The number of floating point operations is directly proportional to the number of points included in the subdomain. Since a parallel application is driven by the most loaded process in the pool, we will evaluate how the number of points changes at different decompositions for the process with the biggest domain considering the two data structures. Figure 9 reports the ratio between the number of points of the biggest domain for the three-dimensional (hence including the land points) and the one-dimensional data structure. For small decompositions (less than 1026) the three-dimensional data structure includes an overhead due to the operations over the 


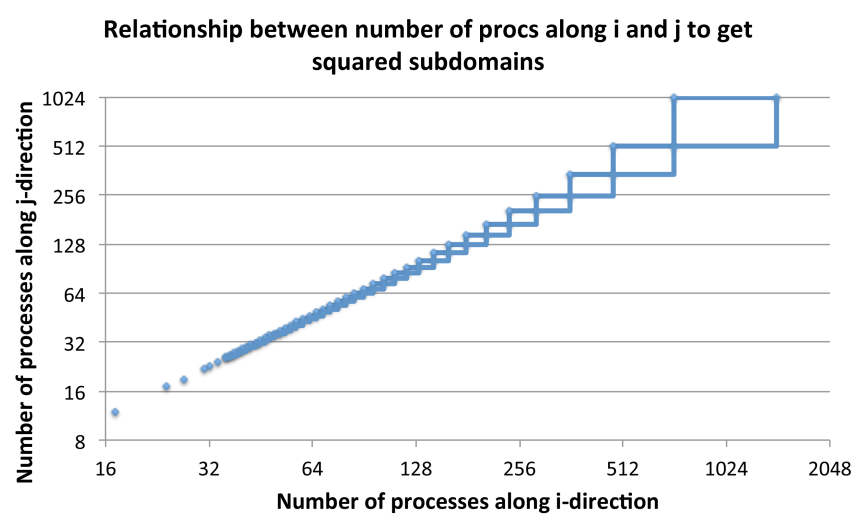

Figure 8. Relationship between the number of processes along the $i$ and $j$ directions to get exactly squared subdomains. If the number of processes falls within the blue boxes, the resulting decomposition is a perfect square.

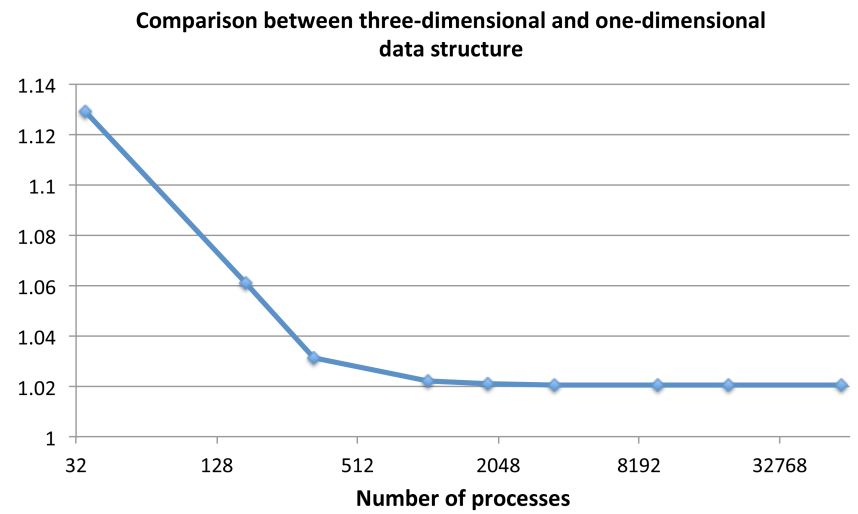

Figure 9. Ratio between the number of floating point operations when using the three-dimensional and one-dimensional data structures.

land points which reaches $12 \%$. When the number of processes increases, even if the subdomains become smaller and the most loaded process should include ocean points only, the three-dimensional approach introduces a $2 \%$ of computational overhead since the last level in the bottom is composed entirely of land points.

\subsection{Load balancing}

The load balancing is measured by evaluating how many points are taken by each process. An optimal load balancing is reached when each process elaborates the same number of points. Even if some alternative and efficient balancing approaches have been proposed for a multi-core aware partitioning of the NEMO domain (Pickles and Porter, 2012), the NEMO v3.4 release is based on a three-dimensional decomposition which equally divides the domain among the processes not considering the number of ocean points in the subdomain. In the case that the domain size is not perfectly divisible by the number of processes (along the $i$ or $j$ direction), some processes have one additional row or column. In this case the work load is well balanced. Figure 10 graphically represents the number of points for each domain. Each square is a process and the colour represents the number of points (the lighter the colour, the lower the number of points). The black squares are those domains made entirely by land points, and they are excluded from the computation. With the one-dimensional data structure the work load balancing is different, as illustrated in Fig. 10. In this case the number of points for each domain depends on the bathymetry; domains near the coast have fewer points, resulting in an unbalanced workload. The workload unbalancing can also be evaluated in Fig. 11, where the histogram of the ocean point distribution for the one-dimensional data structure is reported. Table 6 reports the analytical values and an estimation of how much improvement can be reached with an ideal distribution of the ocean points among the processes. The overhead due to the load balancing ranges from 50 to $30 \%$ of the execution time. Even if the one-dimensional approach is unbalanced, taking into account the considerations made in the previous section and in Fig. 9, the most loaded processes in both approaches have the same number of points (for more than 1026 processes). This implies that the apparently wellbalanced computation given by the three-dimensional data structure does not necessarily lead to improved performance because it is given by an increment of computation by those processes which have few ocean points, and it is not given by a balanced distribution of the useful computation (i.e. the computation performed over the ocean points).

\subsection{Memory allocation}

The BFM is quite sensitive to the amount of allocated memory since it handles tens of state variables. For simulations at high resolution the memory could be a limiting factor. Figure 12 depicts the amount of memory needed by the BFM when using the three- and one-dimensional data structure. The graph reports the increment of memory with respect to the minimum required memory. The amount of memory increases due to the halos: the higher the number of processes, the larger the redundant memory that is needed to store the elements in the halos. This clearly indicates that the threedimensional data structure requires an amount of additional memory estimated between 50 and $120 \%$ for storing the land points. This is one of the principal motivations which suggest that the three-dimensional data structure is not suitable for the BFM.

To conclude, the one-dimensional data structure performs better or, in the worst case, equal to the three-dimensional one in terms of floating point operations. Moreover, the onedimensional data structure requires the minimum amount of memory, since it stores only the ocean points, while the three-dimensional approach increases the amount of memory for a very high factor, demanding a huge amount of mem- 


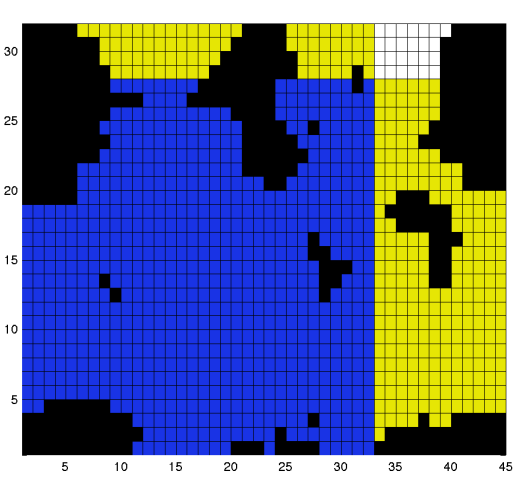

(a) $3 d-1026$ procs

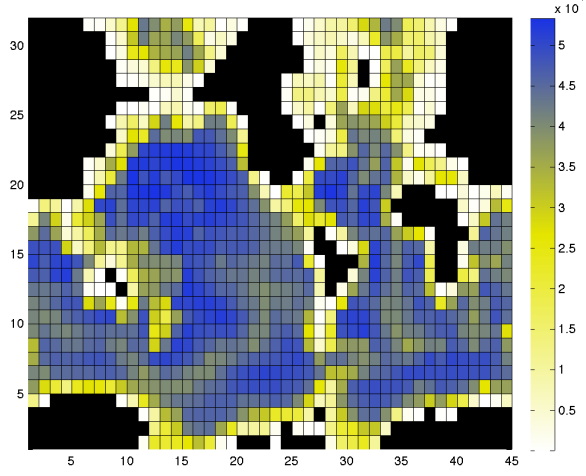

(c) $1 d-1026$ procs

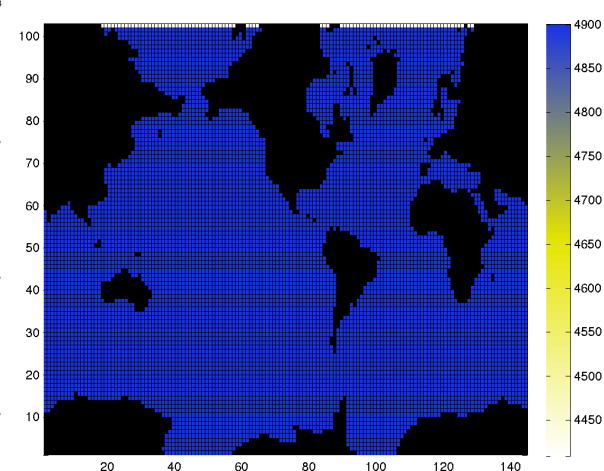

(b) $3 d-9882$ procs

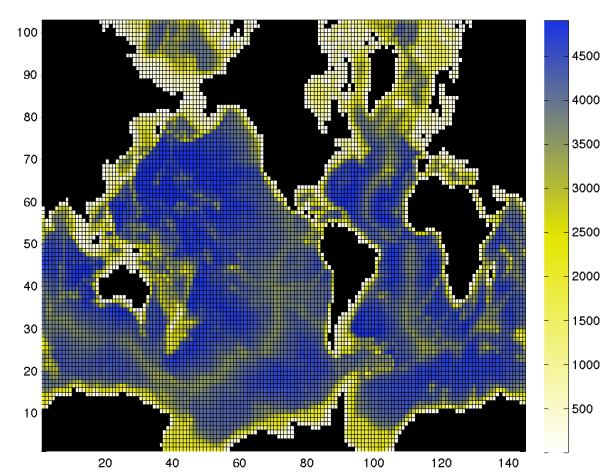

(d) $1 d-9882$ procs

Figure 10. Load balancing for one-dimensional (c, d) and three-dimensional (a, b) data structures with 1026 (a, c) and 9882 (b, d) processors. The colours represent the number of points in the domain.
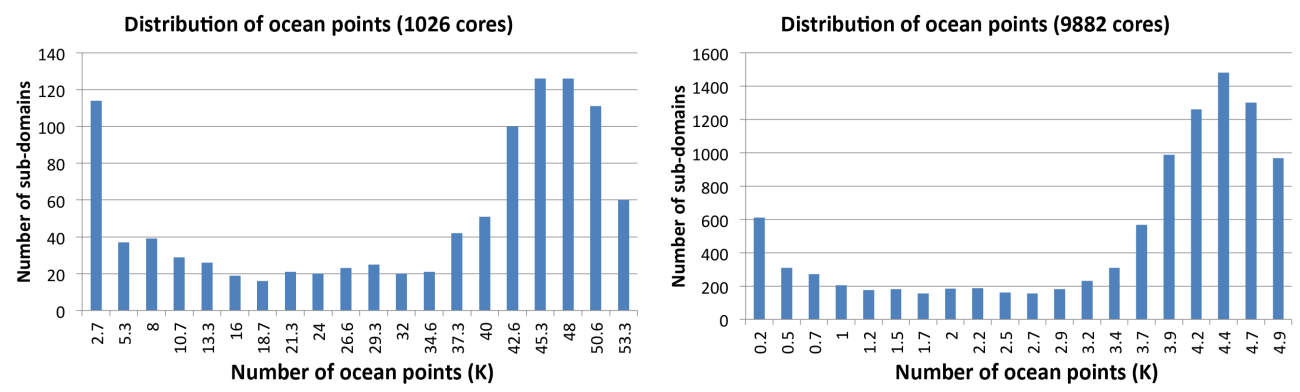

Figure 11. Histogram of the ocean point distribution using the one-dimensional data structure for two different configurations, with 1026 and 9882 processes.

ory and making prohibitive the simulations at high resolution. Finally, even if the workload is not balanced, the solution for a better balancing is not given by the use of the three-dimensional data structure. An ad hoc policy to redistribute the ocean points among the processes could bring ideally a performance improvement of more than $30 \%$. The counterparts are the costs for data remapping between onedimensional and three-dimensional data structures, which occur during the coupling steps between the BFM and NEMO. However, the remapping is not accounted for as an hotspot by the profiler (Sect. 4). Moreover, for a few processes (less than 1026), the penalty due to the remapping is balanced out by the reduction in terms of the number of floating point operations, while for a greater number of processes the remapping can be skipped, since the subdomains are entirely made up of ocean points.

\section{The memory model}

The presence of the BFM component in the coupled model produces a work load unbalancing due to the different numbers of ocean points assigned to processes. We already stated that a better load balancing policy would notably improve the 
Table 6. Load balancing when adopting the three-dimensional or one-dimensional data structure. The first column reports the number of processes followed by the dimension of the biggest domain. The Max and Avg columns report the maximum number of grid points (i.e. the number of grid points for the biggest domain) and the average value among all the domains. The Unbal. columns give the estimation of the overhead due to unbalancing. It is computed as (Max-Avg) / Max.

\begin{tabular}{lrrrrrrrrr}
\hline & & \multicolumn{3}{c}{ Three-dim. data struct. } & & \multicolumn{2}{c}{ One-dim. data struct. } \\
\cline { 3 - 4 } \cline { 8 - 10 } Procs. & Subdomain & Max & Avg & Unbal. & & Max & Avg & Unbal. \\
\hline 35 & $208 \times 206$ & 2142400 & 2137380 & $0.23 \%$ & & 1897483 & 952326 & $49.81 \%$ \\
171 & $87 \times 87$ & 378450 & 376899 & $0.41 \%$ & & 356746 & 200427 & $43.82 \%$ \\
332 & $62 \times 62$ & 192200 & 192032 & $0.09 \%$ & & 186381 & 105147 & $43.59 \%$ \\
1026 & $35 \times 35$ & 61250 & 60653 & $0.98 \%$ & & 59930 & 35848 & $40.18 \%$ \\
1856 & $26 \times 26$ & 33800 & 33524 & $0.82 \%$ & & 33109 & 20680 & $37.54 \%$ \\
3572 & $19 \times 19$ & 18050 & 17998 & $0.29 \%$ & & 17689 & 11436 & $35.35 \%$ \\
9882 & $12 \times 12$ & 7200 & 7195 & $0.07 \%$ & & 7056 & 4764 & $32.48 \%$ \\
19745 & $9 \times 9$ & 4050 & 4039 & $0.27 \%$ & & 3969 & 2738 & $31.01 \%$ \\
59955 & $6 \times 6$ & 1800 & 1771 & $1.64 \%$ & & 1764 & 1233 & $30.13 \%$ \\
\hline
\end{tabular}

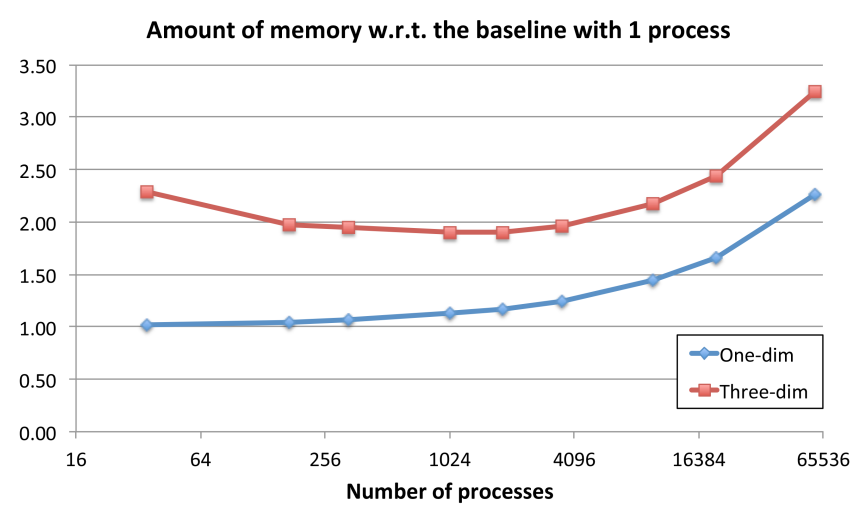

Figure 12. Amount of memory allocated using three- and onedimensional data structures. The values refer to the minimum amount of memory allocated in a sequential run.

performance, even though an optimal mapping of the processes over the computing nodes can bring a slight improvement without changing the application code. The load unbalancing affects both the number of floating point operations and also the amount of memory allocated by each process. The local resource manager of a parallel cluster (LSF - Load Sharing Facility, PBS - Portable Batch System, etc.) typically handles the execution of a parallel application mapping the processes on the cores of each computing node without any specific criteria, just following the cardinal order of the MPI ranks. This generates an unbalanced allocation of memory on the nodes; some nodes can saturate the main memory and some others could use only a small part of it. The amount of allocated memory is also an indirect measurement of the memory accesses, as the larger the allocated memory the higher the number of memory accesses. For those nodes with full memory allocation, the memory contention among the processes impacts on the overall performance. A fairer distribution of processes over the computing nodes can bet- ter balance the allocated memory, reducing the memory contention. In this section we describe a mathematical model to estimate the amount of memory required by each process. The memory model can be used to choose an optimal domain decomposition (i.e. a decomposition such that the memory footprint of the heaviest process is minimum) or it can be used to evenly map each process on computational nodes using the amount of memory per node as a criterion.

The model was built considering the peculiarities of the data structures used in NEMO and the BFM as discussed in the previous section. In general, the memory allocated by each process is given by a term directly proportional to the subdomain size (according to the data allocated in NEMO), a term directly proportional to the number of ocean points in the subdomain (according to the data allocated in BFM) and a constant quantity of memory related to the scalar variables and the data needed for parallel processes management.

The memory model can be formalised by the following equation:

$M=\alpha \times \mathrm{jpi} \times \mathrm{jpj} \times \mathrm{jpk}+\beta \times \mathrm{Opt}+\gamma$,

where jpi, jpj and jpk represent the size of the subdomain along the three dimensions and Opt is the number of ocean points in the subdomain. As in a linear model we can evaluate the coefficients $\alpha, \beta$ and $\gamma$ using a linear regression.

The test configuration used to evaluate the coefficients is executed on 672 processes and, for each one, the total amount of allocated memory was measured. The job_memusage tool, developed by the CISL Consulting Services Group at UCAR, has been used to measure the total (peak) memory use for each process. The number of ocean points of each subdomain is evaluated using the bathymetry input file. Figure 13 shows the memory evaluated for the configuration with 672 processes on the Sandy Bridge architecture. The data reported in Fig. 13 also show that processes that have a very similar number of ocean points may require quite different amounts of memory. This amount of memory does 


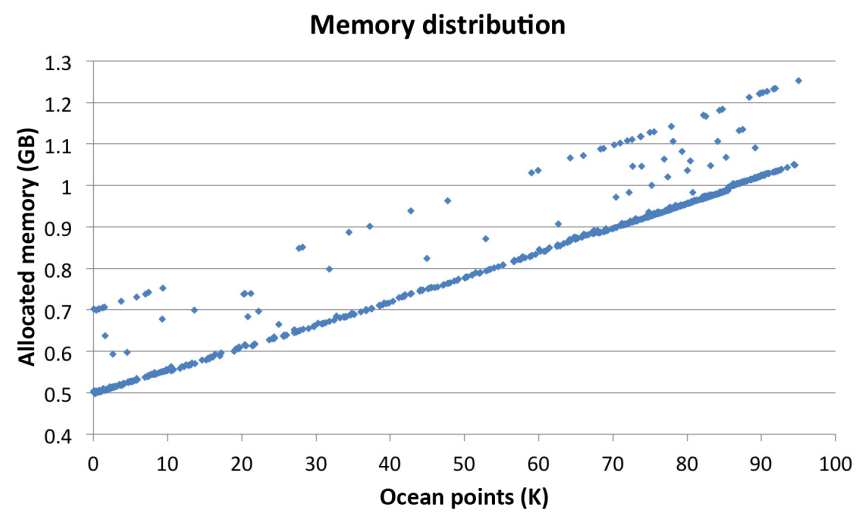

Figure 13. The relationship between the number of ocean points belonging to a subdomain and the memory footprint needed to process that subdomain. The chart shows the data extracted from a reference run on 672 processes (hence 672 subdomains) on the ATHENA cluster. The data have been used to evaluate the memory model coefficients.

not depend either on the number of ocean points or on the model itself since, with different executions of the same configuration, a given process may require significantly different amounts of memory. Probably it is due to some memory allocation at system call level. This deserves further investigation.

Table 7 reports the evaluation of the coefficients obtained with the linear regression, the standard error and the coefficient of determination $\left(R^{2}\right)$, which refers to the difference between the value of memory estimated and measured. It can assume values between 0 and 1 . A value of 1 means that there is a perfect correlation, i.e. there is no difference between the estimated value and the actual one. The memory model has been validated with other domain decompositions ranging from 160 to 512 cores (see Fig. 14 as an example of comparison between the memory measured for each process and the estimation from the memory model). A detailed evaluation of the memory model accuracy is reported in Table 8 . It shows the value of the root mean square error (RMSE), expressed in GigaBytes, for each examined decomposition. The relative RMSE, instead, expresses the ratio between the root mean square error and the average of the examined sample. The relative RMSE is always less than $6 \%$, so we can assume that the memory model estimates the actual trend with a good approximation.

Figure 15 shows the trend of the memory footprint estimated by the model. The difference between the processes with the most allocated memory (red line) and the least allocated memory (blue line) also gives a measure of the load unbalancing, which is greater for the smallest decompositions and decreases (i.e. the computation is better balanced) for the highest decompositions. This can be explained since the highest decompositions give smaller subdomains with a number of land points evenly distributed (recall that the
Table 7. Estimation of the memory model coefficients. The evaluation has been experimentally performed considering a decomposition made up of $19 \times 45$ subdomains, with 183 of them having land points only (672 parallel processes have been used for the simulation).

\begin{tabular}{lr}
\hline Coefficient & Value \\
\hline$\alpha$ & $1.030 \mathrm{~KB}$ \\
$\beta$ & $6.142 \mathrm{~KB}$ \\
$\gamma$ & $421.44 \mathrm{MB}$ \\
$R^{2}$ & $97.49 \%$ \\
Standard error & $62.62 \mathrm{MB}$ \\
\hline
\end{tabular}

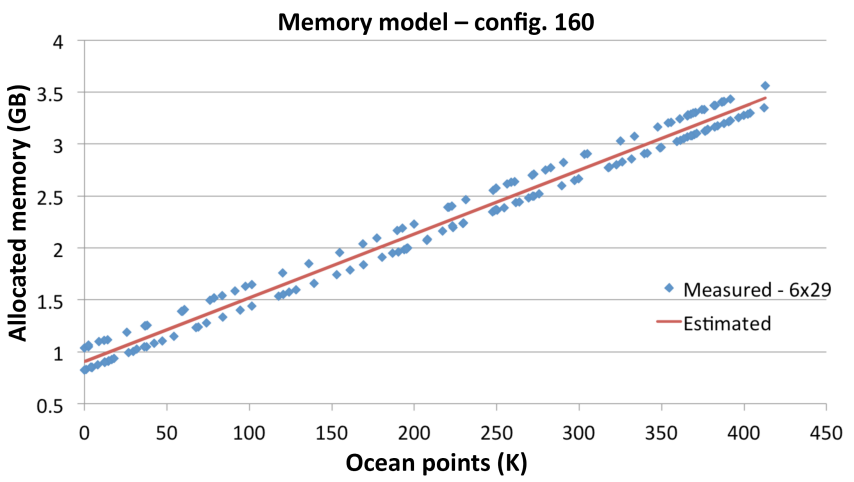

Figure 14. Comparison between the memory model trend (red line) and the experimental values (blue line) for a reference configuration on 160 processes. The decomposition is made up of $6 \times 29$ subdomains, where 14 of them are with land points only.

Table 8. Evaluation of the memory model accuracy. The first column reports the examined decompositions, the last one shows the root mean square error (RMSE), expressed in GigaBytes, while the second one shows the relative RMSE expressed as the root mean square error compared with the average of the examined sample.

\begin{tabular}{lrr}
\hline Configuration & Relative RMSE (\%) & RMSE (GB) \\
\hline 160 & 4.651 & 0.0995 \\
192 & 5.106 & 0.0950 \\
224 & 4.647 & 0.0763 \\
256 & 5.489 & 0.0813 \\
288 & 5.773 & 0.0790 \\
320 & 5.568 & 0.0698 \\
512 & 4.907 & 0.0473 \\
\hline
\end{tabular}




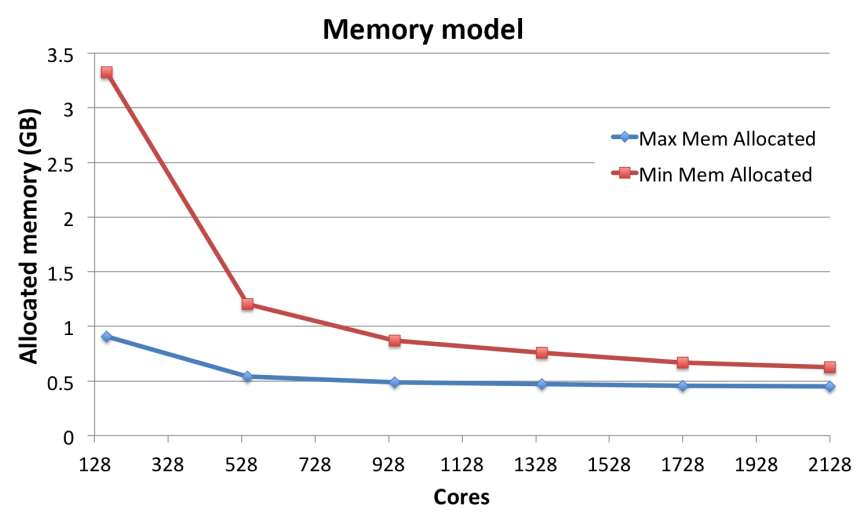

Figure 15. Estimation of the memory footprint using the memory model for an increasing number of processes. The red and blue lines respectively indicate the maximum and minimum allocated memory among the processes involved.

subdomains with land points only are excluded from the computation). This test also shows that in a smaller configuration the memory required by each process is substantially larger, and then it is more likely to have an additional time overhead due to the combination of processes on a node requesting more memory than the one available.

\section{Conclusions}

The present work aimed at analysing the computational performance of the PELAGOS coupled model at $0.25^{\circ}$ of horizontal resolution on two different computing architectures, in order to identify the presence of computational bottlenecks and limiting factors to the scalability on many core architectures. The analysis highlighted three main aspects limiting the model scalability.

- The I/O management. Before starting the scalability analysis, some tests on the two architectures have been performed using the model complete with all of its features. The management of I/O is inefficient when the number of processes increases. In fact, the number of reading/writing files is proportional to the number of processes. On the one hand this peculiarity allows the parallelisation of the $\mathrm{I} / \mathrm{O}$ operations (each process can read/write its inputs/outputs independently); on the other hand, the I/O management is prohibitive when we have thousands of processes. For this reason, the I/O has been omitted from the performance analysis, focusing only on the computational aspects. In future, the adoption of a more performant I/O strategy will be necessary (e.g. the use of the XIOS tool for I/O management).

- The memory usage balancing. The presence of the BFM component introduces a load imbalance due to the different number of ocean points belonging to each subdomain. Since the memory allocated by each process is related to the number of ocean points, a balancing strategy of the memory allocated for each node would improve the performance. In this context, some mapping strategies of the processes on the physical cores could be taken into account.

- The communication overhead. PELAGOS is based on a pure MPI parallelisation. When the number of processes increases, the ratio between computation and communication decreases. Beyond a limit, the communication overhead becomes unsustainable. A possible solution is to parallelise along the vertical direction or overlap communications with computation. A hybrid parallelisation strategy can be taken into account, adding for example OpenMP to MPI. This strategy would allow a better exploitation of many-core architectures. Moreover, a further level of parallelism over the state variables treated by the BFM could be introduced.

The work has also demonstrated that the one-dimensional data structure used in the BFM does not affect the performance when compared with the three-dimensional data structure used in NEMO. The workload in the BFM is unbalanced since the global domain is divided among the processes following a block decomposition without taking into account the number of ocean points which fall in a subdomain. The adoption of smarter domain decomposition, e.g. based on the number of ocean points, could lead to a significant improvement in the performance at lower process counts. When the number of processing elements is greater than 1024 , the difference between both strategies is negligible (see Fig. 9).

Finally, the current version of PELAGOS025 is still far from being ready for scaling on many-core architecture. A constructive collaboration between computational scientists and application domain scientists is a key step to reaching substantial improvements toward the full exploitation of next-generation computing systems.

\section{Code availability}

The PELAGOS025 software is based on NEMO v3.4 and BFM v5.0, both available for download from the respective distribution sites (http://www.nemo-ocean.eu/ and http:// bfm-community.eu/). The software for coupling NEMO v3.4 with BFM v5.0 is available upon request (please contact the BFM system team - bfm_st@lists.cmcc.it). Section 3 of the BFM manual (Vichi et al., 2015a) reports all the details on the coupling. Finally, the ORCA025 configuration files used for this work are available upon request to the paper authors.

Acknowledgements. The authors thankfully acknowledge the computer resources, technical expertise and assistance provided by the Argonne Leadership Computing Facilities, namely Paul Messina. The authors acknowledge the NEMO and BFM consortia for the use of the NEMO system and the BFM system. 
This work was partially funded by the EU Seventh Framework Programme within the IS-ENES project (grant number 228203) and by the Italian Ministry of Education, University and Research (MIUR) with the GEMINA project (grant number DD 232/2011).

Edited by: A. Ridgwell

\section{References}

Balaji, V., Redler, R., and Budich, R. G. P. (Eds.): Earth System Modelling Volume 4: IO and Postprocessing, Springer, Berlin, Heidelberg, Germany, published online, 2013.

Balay, S., Gropp, W. D., McInnes, L. C., and Smith, B. F.: Efficient management of parallelism in object oriented numerical software libraries, in: Modern Software Tools in Scientific Computing, edited by: Arge, E., Bruaset, A. M., and Langtangen, H. P., Springer Science and Business Media, LLC, 163-202, Boston, Massachusetts, USA, 1997.

Barnier, B., Madec, G., Penduff, T., Molines, J., Treguier, A., Le Sommer, J., Beckmann, A., Biastoch, A., Boening, C., Dengg, J., Derval, C., Durand, E., Gulev, S., Remy, E., Talandier, C., Theetten, S., Maltrud, M., McClean, J., De Cuevas, B. S., Morales Maqueda, M. Á., Legat, V., and Fichefet, T.: Impact of partial steps and momentum advection schemes in a global ocean circulation model at eddy-permitting resolution, Ocean Dynam., 56, 543-567, 2006.

Blackford, L. S., Choi, J., Cleary, A., Demmel, J., Dhillon, I., Dongarra, J., Hammarling, S., Henry, G., Petitet, A., Stanley, K., Walker, D., and Whaley, R. C.: ScaLAPACK: a portable linear algebra library for distributed memory computers - design issues and performance, in: Proceedings of the 1996 ACM/IEEE conference on Supercomputing, 17-22 November 1996, Pittsburgh, Pennsylvania, USA, 5 pp., 1996.

Claussen, M.: Earth system models, in: Understanding the Earth System: Compartments, Processes and Interactions, edited by: Ehlers, E. and Krafft, T., Springer, Heidelberg, Berlin, Germany, New York, USA, 147-162, 2000.

Dennis, J. M. and Loft, R. D.: Refactoring scientific applications for massive parallelism, in: Numerical Techniques for Global Atmospheric Models, Lecture Notes in Computational Science and Engineering, edited by: Lauritzen, P., Jablonowski, C., Taylor, M., and Nair, R., Springer, Berlin, Heidelberg, Germany, 539-556, 2011.

Dongarra, J., Du Croz, J., Hammarling, S., and Hanson, R. J.: An extended set of FORTRAN basic linear algebra subprograms, ACM T. Math. Software, 14, 1-17, doi:10.1145/42288.42291, 1988.

Dongarra, J., Du Croz, J., Hammarling, S., and Duff, I. S.: A set of level 3 basic linear algebra subprograms, ACM T. Math. Software, 16, 1-17, doi:10.1145/77626.79170, 1990.

Dongarra, J., Beckman, P., Moore, T., et al.: The International Exascale Software Project roadmap, Int. J. High Perform. C., 25, 3-60, doi:10.1177/1094342010391989, 2011.

Epicoco, I., Mocavero, S., and Aloisio, G.: A performance evaluation method for climate coupled models, in: Proceedings of the 2011 International Conference on Computational Science (ICCS), 1-3 June 2011, Singapore, 1526-1534, 2011.

Epicoco, I., Mocavero, S., Macchia, F., and Aloisio, G.: The roofline model for oceanic climate applications, in: Proceedings of Inter- national Conference on High Performance Computing \& Simulation (HPCS), 21-25 July 2014, Bologna, Italy, 732-737, doi:10.1109/HPCSim.2014.6903762, 2014.

Lakner, G., Chung, I. H., Cong, G., Fadden, S., Goracke, N., Klepacki, D., Lien, J., Pospiech, C., Seelam, S. R., and Wen, H. F.: IBM System Blue Gene Solution: Performance Analysis Tools, IBM Redpaper Publication, available at: http://ibm. com/redbooks (last access: 8 June 2016), 2008.

McKiver, W., Vichi, M., Lovato, T., Storto, A., and Masina, S.: Impact of increased grid resolution on global marine biogeochemistry, J. Marine Syst., 147, 153-168, 2015.

Mirin, A. A. and Worley, P. H.: Improving the performance scalability of the community atmosphere model, Int. J. High Perform. C., 26, 17-30, doi:10.1177/1094342011412630, 2012.

Parashar, M., Li, X., and Chandra, S.: Advanced Computational Infrastructures for Parallel and Distributed Applications, Vol. 66, John Wiley and Sons, Hoboken, New Jersey, USA, 2010.

Pickles, S. M. and Porter, A. R.: Developing NEMO for Large Multi-core Scalar Systems, Technical Report of the dCSE NEMO project, DL-TR-2012-00, available at: https://epubs.stfc.ac.uk/work/63488 (last access: 8 June 2016), 2012.

Reid, F. J. L.: NEMO on HECToR - A dCSE Project, Report from the dCSE project, EPCC and University of Edinburgh, UK, 2009.

Schellnhuber, H. J.: Earth system analysis and the second Copernican revolution, Nature, 402, C19-C23, 1999.

Siedler, G., Griffies, S. M., Gould, J., and Church, J. A.: Ocean Circulation and Climate: A 21st century perspective, Vol. 103, Academic Press, Amsterdam, the Netherlands, 2013.

Vichi, M. and Masina, S.: Skill assessment of the PELAGOS global ocean biogeochemistry model over the period 1980-2000, Biogeosciences, 6, 2333-2353, doi:10.5194/bg-6-2333-2009, 2009.

Vichi, M., Pinardi, N., and Masina, S.: A generalized model of pelagic biogeochemistry for the global ocean ecosystem. Part I: Theory, J. Marine Syst., 64, 89-109, 2007.

Vichi, M., Gutierrez Mlot, G. C. E., Lazzari, P., Lovato, T., Mattia, G., McKiver, W., Masina, S., Pinardi, N., Solidoro, C., and Zavatarelli, M.: The Biogeochemical Flux Model (BFM): Equation Description and User Manual, BFM version 5.0 (BFM-V5), Release 1.0, BFM Report Series 1, Bologna, Italy, 2015a.

Vichi, M., Lovato, T., Gutierrez Mlot, E., and McKiver, W.: Coupling BFM with ocean models: the NEMO model (Nucleus for the European Modelling of the Ocean), Release 1.0, BFM Report Series 2, Bologna, Italy, doi:10.13140/RG.2.1.1652.6566, 2015b.

Washington, W. M.: The computational future for climate change research, J. Phys. Conf. Ser., 16, 317-324, doi:10.1088/17426596/16/1/044, 2005.

Washington, W. M.: Scientific grand challenges: challenges in climate change science and the role of computing at the extreme scale, Report from the DOE Workshop, 6-7 November 2008, Washington D.C., USA, 2008.

Worley, P. H., Craig, A. P., Dennis, J. M., Mirin, A. A., Taylor, M. A., and Vertenstein, M.: Performance and performance engineering of the community Earth system model, in: Proceedings of the 2011 ACM/IEEE Conference on Supercomputing, 12-18 November 2011, Seattle, WA, USA, Article 54, 2011.

XIOS: XIOS wiki page, available at: http://forge.ipsl.jussieu.fr/ ioserver/, last access: 2 December 2013. 